\title{
Effect of velocity uncertainty on migrated reflectors
}

Oleg V. Poliannikov* and Alison E. Malcolm

Earth Resources Laboratory, Department of Earth, Atmospheric, and Planetary Sciences, MIT

\section{SUMMARY}

We study the problem of uncertainty quantification for migrated images. A traditional migrated image contains deterministic reconstructions of subsurface structures. However, the input parameters used in migration, such as reflection data and a velocity model, are inherently uncertain. This uncertainty is carried through to the migrated images. We use Bayesian analysis to quantify the uncertainty of migrated structures by constructing a joint statistical distribution of the structures in question. From this distribution we can deduce the uncertainty in absolute positions of the structures, or a relative position of one structure with respect to another. In some cases the relative position has a much smaller uncertainty than the absolute position.

\section{INTRODUCTION}

Seismic depth-migration is a general process by which seismic events in recorded data are moved from time to depth coordinates (Yilmaz, 2001). This process traditionally produces a single image. Any subsequent interpretation is based on this image as if it is an accurate representation of the subsurface. In order to go from time to space, any implementation of depth migration requires a velocity model. This velocity model is obtained from prior surveys and analysis, and it is assumed given for the purpose of migration. Obtaining an accurate velocity model in practice is a non-trivial problem. The final model is an approximation to the true model that can be associated with some uncertainty. This uncertainty propagates to the migrated image as a whole, and to the locations of individual horizons in particular (Grubb et al., 2001; Pon and Lines, 2005; Glogovsky et al., 2009; Osypov et al., 2011). The Bayesian uncertainty analysis that we advocate in this paper recovers the absolute and relative positions of seismic horizons along with their associated uncertainty. It follows a similar analysis of location uncertainty for seismic events (Poliannikov et al., 2014). Given surface seismic reflection data, we construct a joint posterior estimator of the locations of chosen horizons that is a multidimensional probability distribution of discrete points on the horizons. This probability distribution describes the location of each horizon as well as the correlation between the locations of different horizons. It is derived from a prior probability of the horizon locations, and the likelihood function that describes the probability of observing a particular dataset given fixed horizon locations. This likelihood function is based on map demigration, which is analytic and therefore fast. Our analysis shows that in some cases the location of one structure relative to another can be known much better than the absolute depth of individual structures, and this reduction of uncertainty can be exactly quantified. This analysis is applicable in many situations from redatuming below a complicated near-surface to updating the model during monitoring-whiledrilling.

\section{THEORY}

Problem setup

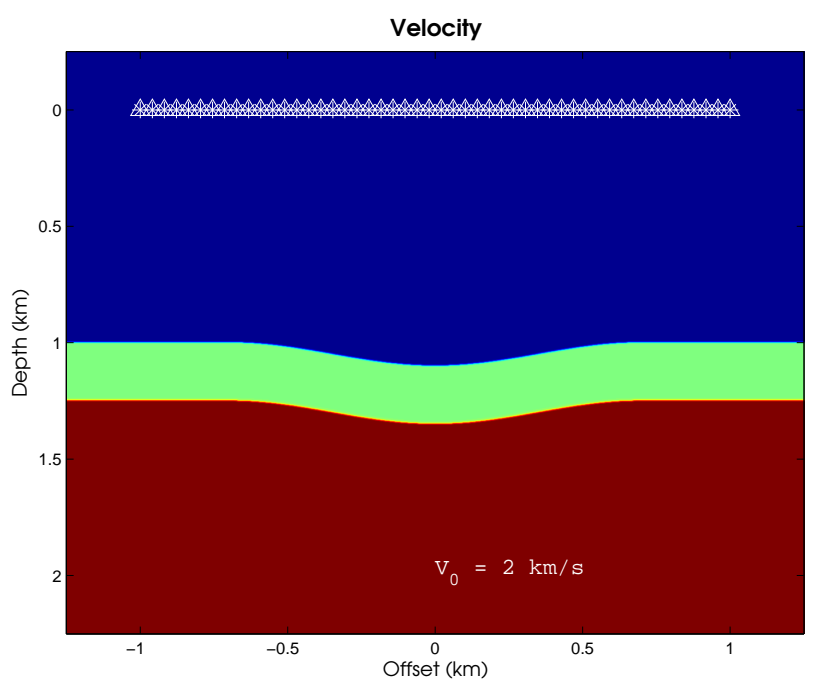

Figure 1: A numerical acoustic model with two reflectors, and sources and receivers at the surface. The part of the medium above the first reflector $(z=1 \mathrm{~km})$ is uncertain in later examples. The velocity below the reflector $z \geq 1$ is assumed known.

We consider a simple acoustic model with two reflectors embedded in it. The velocity model is uncertain. We model the velocity uncertainty by assuming that the velocity, $V$, belongs to a family of admissible velocity models, $\mathscr{V}$ The probability distribution, $p(V)$, determines the likelihood of any velocity model from the family, $\mathscr{V}$. Sources and receivers are located at the surface as shown in Figure 1. For each source location, we fire a shot and record a corresponding common-shot gather. This common-shot gather can then be resorted, e.g., to common-offset gathers, and individual reflections can be picked in the sorted gather. The problem is to describe the location and shape of the reflectors along with the associated uncertainty from those reflected events in the gathers. The current discussion relies on the picking of event travel-times and migrates those picked times directly. This is, of course, not ideal for a real data set. In that case, we would expand our data in a suitable basis, e.g., curvelets or similar, and migrate the time indices of these bases functions. This is a subject of current work.

\section{Map migration}

For a $2 \mathrm{D}$ survey in a $3 \mathrm{D}$ space, each event in a common- 


\section{Effect of velocity uncertainty on migrated reflectors}

offset gather can be approximated with a collection of fivetuples $\left(x_{u}, y_{u}, t_{u}, p_{u}^{x}, p_{u}^{y}\right)$, where $x_{u}, y_{u}$ are common-midpoint coordinates, $t_{u}$ is the travel time, and $p_{u}^{x}=1 / 2 \partial t_{u} / \partial x_{u}, p_{u}^{y}=$ $1 / 2 \partial t_{u} / \partial y_{u}$ are the horizontal slownesses of the un-migrated reflection. For simplicity we will work in $2 \mathrm{D}$, where $y_{u} \equiv 0$ and $p_{u}^{y} \equiv 0$, and the event in the data domain has the form $\left(x_{u}, t_{u}, p_{u}^{x} \equiv p_{u}\right)$. All results, however, generalize directly to $3 \mathrm{D}$. For a homogeneous isotropic medium with velocity $V$, the travel time is governed by the double-square-root equation:

$$
\begin{aligned}
t_{u} & =\frac{1}{V} \sqrt{\left(x_{u}-x_{m}-h\right)^{2}+\left(\frac{V t_{m}}{2}\right)^{2}} \\
& +\frac{1}{V} \sqrt{\left(x_{u}-x_{m}+h\right)^{2}+\left(\frac{V t_{m}}{2}\right)^{2}} .
\end{aligned}
$$

Here $x_{m}$ is the coordinate of the reflection point, and $t_{m}$ is the two-way migrated travel time. Defining the local dip of the reflector, $p_{m}=1 / 2 \partial t_{m} / \partial x_{m}$, we obtain the migrated reflector coordinates $\left(x_{m}, t_{m}, p_{m}\right)$ Douma and de Hoop (2006) derive an analytic relationship between the migrated reflector coordinates $\left(x_{m}, t_{m}, p_{m}\right)$ and the specular reflection coordinates $\left(x_{u}, t_{u}, p_{u}\right)$ It has the following form:

$$
\begin{gathered}
x_{m}=x_{u}-\left(\frac{V t_{u}}{2}\right)^{2} \frac{\Lambda_{u}}{h} \\
t_{m}=2\left(\frac{t_{u}^{2}}{4}-\frac{h^{2}}{V^{2}}+\left(\frac{V t_{u} \Lambda_{u}}{4 h}\right)^{2}\left(\frac{4 h^{2}}{V^{2}}-t_{u}^{2}\right)\right)^{2} \\
p_{m}=\frac{1}{2} p_{u} t_{u}\left|\Lambda_{u}-1\right|\left|\Lambda_{u}+1\right| \\
\times\left(\frac{t_{u}^{2}}{4}-\frac{h^{2}}{V^{2}}+\left(\frac{V t_{u} \Lambda_{u}}{4 h}\right)^{2}\left(\frac{4 h^{2}}{V^{2}}-t_{u}^{2}\right)\right)^{-\frac{1}{2}}
\end{gathered}
$$

where

$$
\begin{gathered}
\Lambda_{u}=\frac{1}{2 \sqrt{2} p_{u} h} \sqrt{\Theta_{u}\left(1-\sqrt{1-\frac{64\left(p_{u} h\right)^{4}}{\Theta_{u}^{2}}}\right)} \\
\Theta_{u}=t_{u}^{2}+\left(\frac{2 h}{V}\right)^{4} \frac{1}{t_{u}^{2}}-2\left(\frac{2 h}{V}\right)^{2}\left(1-\left(V p_{u}\right)^{2}\right) .
\end{gathered}
$$

The demigration equations describe the transformation back from $\left(x_{m}, t_{m}, p_{m}\right)$ to $\left(x_{u}, t_{u}, p_{u}\right)$. Formulas for this transformation are given by Douma and de Hoop (2006) and are not repeated here.

\section{Bayesian uncertainty analysis}

Forward model

If the velocity, $V$, is known and there is no noise in the data, then the reflector can be represented in the depth domain as a triplet $\left(x_{m}, t_{m}, p_{m}\right)$ or in the data domain as a triplet $\left(x_{d}, t_{d}, p_{d}\right)$. These two representations are related through the operators of map migration, $\mathscr{M}$, and map demigration, $\mathscr{D}$ :

$$
\left(x_{d}, t_{d}, p_{d}\right) \equiv \ell_{d} \stackrel{\mathscr{M}}{\rightarrow} \ell_{m} \equiv\left(x_{m}, z_{m}, p_{m}\right),
$$

and

$$
\left(x_{m}, t_{m}, p_{m}\right) \equiv \ell_{m} \stackrel{\mathscr{D}}{\rightarrow} \ell_{d} \equiv\left(x_{d}, z_{d}, p_{d}\right) .
$$

In practice, the velocity model is never known exactly, and the recorded data is noisy. Velocity randomness and the noise in the recordings make the travel time data random, and the problem becomes that of recovering the model for the subsurface from those random observations. We solve this problem by Bayesian inversion. The Bayesian framework is based on the notion of the likelihood function. Consider a model that consists of reflectors, $\left(x_{m}, t_{m}, p_{m}\right)$, encoded in the common-offset space as well as an assumed model, $V$, and the offset, $h$. The observed data is then written as

$$
\hat{t}_{d}=t_{d}+n_{t},
$$

where $\left(x_{d}, t_{d}, p_{d}\right)=\mathscr{D}\left(x_{m}, t_{m}, p_{m} \mid V, h\right)$. Here we explicitly indicate the velocity and offset used in the demigration operator We assume that there is no noise in the $x_{d}$ component, i.e., $\hat{x}_{d}=x_{d}$, because the travel times are registered at fixed receiver locations. The noise $n_{t}$ is assumed to be a realization of a zero-mean Gaussian process with some correlation length $\ell$. If reflection times were picked independently at each receiver, the correlation length would be zero: $\ell=0$. However, because in practice events are picked in the entire gather, where the pick in any given trace depends on the signal content at neighboring traces, the correlation length is greater than zero: $\ell>0$ The noisy slownesses $p_{d}$ are estimated from $\hat{x}_{d}$ by a finite-difference approximation.

Inversion in known velocity

Denote the likelihood function that describes possible observed data and their probability as

$$
p\left(\hat{x}_{d}, \hat{t}_{d}, \hat{p}_{d} \mid x_{m}, t_{m}, p_{m}, h, V\right) .
$$

The Bayes' formula yields the following inversion:

$$
\begin{aligned}
& p\left(x_{m}, t_{m}, p_{m} \mid \hat{x}_{d}, \hat{t}_{d}, \hat{p}_{d}, h, V\right) \\
& \propto p\left(\hat{x}_{d}, \hat{t}_{d}, \hat{p}_{d} \mid x_{m}, t_{m}, p_{m}, h, V\right) p\left(x_{m}, t_{m}, p_{m}\right)
\end{aligned}
$$

Equation 10 provides an exact expression for the posterior distribution of the model parameters. However, if each reflector is approximated relatively finely, then the total number of model parameters is very large. In order to simplify the computation and the representation of the posterior distribution of the horizon locations, we approximate it with a multi-variate Gaussian distribution,

$$
\left(x_{m}, t_{m}\right) \sim \mathscr{N}\left(\left(x_{m}^{0}, t_{m}^{0}\right), \Sigma^{0}\right) .
$$

Following standard Gaussian analysis, the mean, $\left(x_{m}^{0}, t_{m}^{0}\right)$, and the covariance matrix, $\Sigma^{0}$, of the joint Gaussian distribution are found as follows. The mean is found by solving a constrained maximization problem:

$$
t_{m}^{0}=\arg \max p\left(\hat{x}_{d}, \hat{t}_{d}, \hat{p}_{d} \mid x_{m}, t_{m}, p_{m}, h, V\right) p\left(x_{m}, t_{m}, p_{m}\right)
$$

subject to the constraints

$$
\begin{aligned}
& \hat{x}_{d}=x_{d}, \\
& \hat{p}_{d}=\frac{1}{2} \frac{\partial \hat{d}_{d}}{\partial x_{d}} .
\end{aligned}
$$

The local estimate of the covariance about the mean is given by the inverse hessian of the logarithm of the function inside the arg max in Equation 12 at its maximum point. 


\section{Effect of velocity uncertainty on migrated reflectors}

\section{Inversion in uncertain velocity}

Equations 10 and 11 provide expressions for migrated reflector coordinates in a known velocity model for a fixed offset. When the velocity is uncertain, we can integrate the product of the likelihood function and the prior to obtain the velocity independent posterior. We also integrate over all available offsets assuming the picking errors in each common-offset gather are independent. We thus obtain

$$
\begin{aligned}
& p\left(x_{m}, t_{m}, p_{m} \mid \hat{x}_{d}, \hat{t}_{d}, \hat{p}_{d}, h, V\right) \\
& \propto \mathrm{E}_{V} \int p\left(\hat{x}_{d}, \hat{t}_{d}, \hat{p}_{d} \mid x_{m}, t_{m}, p_{m}, h, V\right) p\left(x_{m}, t_{m}, p_{m}\right) d h
\end{aligned}
$$

The formal expectation in Equation 14 can be approximated with a mean over a sample from the velocity distribution. If the family of admissible velocities, $\mathscr{V}$, is large and multi-dimensional then producing sample velocities from this distribution may be a non-trivial problem. It could do be done, for example through an exploration of velocity models that flatten angle gathers to within a given tolerance. We will not address the problem of velocity sampling in greater detail here. In the examples in the next section we will look at a simple illustration for the proposed methodology, where the numerical computation of the expectation over different velocity models, $V$, is easy.

\section{NUMERICAL EXAMPLES}

We illustrate the proposed methodology with simple numerical examples. The model is as described before with sources and receivers at the surface and two reflectors in the subsurface (Figure 1). The velocity is uncertain, and the recorded signals are noisy. The noise in the signal leads to erroneous picking of the specular reflection events. The correlation length is taken to be, $\ell=1 \mathrm{~km}$, and the standard deviation is $1 \mathrm{~ms}$. We now consider two cases of velocity uncertainty. In both cases the velocity above the reflectors is uncertain, and the velocity below the first reflector is presumed known. This is a simplistic model for a geology frequently encountered in Middle East, where karsts and dunes sit on top of a layered cake of slowly varying sediments. We do not model lateral variations in the velocity model that are certainly very important in practice.

\section{Precision of relative imaging}

In the first example (Figure 2), the velocity estimate in the "overburden" is unbiased but it has an uncertainty of $\pm 2 \%$. We compute the posterior distribution for both reflectors according to Equation 14. In the left panel we plot in solid blue the mean positions of each reflector computed from the posterior distribution. We also compute the offset-dependent standard deviations, $\sigma$, of the depth of each reflector, and indicate the boundaries of the $3 \sigma$ intervals with dashed red lines. In the right panel, we show the relative depth of the second reflector with respect to the depth of the first reflector. A comparison of the panels reveals a marked reduction in uncertainty in relative depth compared to the absolute depth. The relative imaging is more stable because much of the velocity uncertainty has little effect on the relative depth. The relative depth primarily

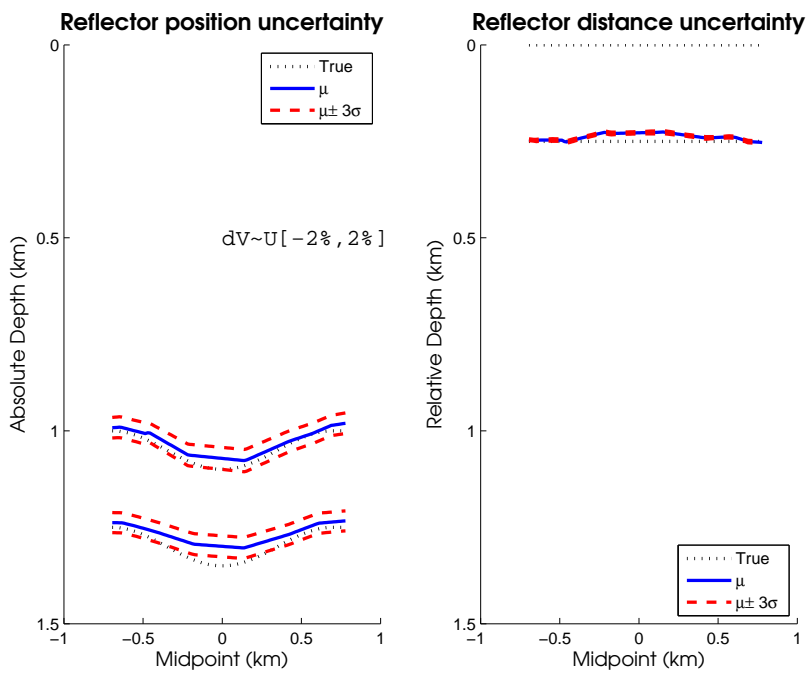

Figure 2: Left: Variations in the position of the migrated reflectors when the overburden velocity is uncertain: $V=$ $V_{0}+d V$, where $d V \sim$ Uniform $[-2 \%, 2 \%]$. Blue lines denote the estimated mean position, and the red dashed lines outline the 3-standard-deviation region plotted with vertical exaggeration to show reflector shape. Right: The relative depth of the second reflector with respect to the depth of the first one. The effect of the velocity uncertainty is much smaller.

depends on the the difference of reflection times of both reflectors. This travel time difference is not significantly affected by the velocity variations in the overburden.

\section{Accuracy of relative imaging}

In the second example (Figure 3), we assume that the velocity prior is biased. More specifically, let the velocity in the overburden above the first reflector be underestimated by a random factor that is distributed uniformly between $1 \%$ and $5 \%$. The velocity between the reflectors is assumed to be known. As before, the left panel contains the results of migrating data in the incorrect velocity. The depths of both reflectors are underestimated due to the low velocity used for migration. However, the relative depth of the second reflector with respect to the depth of the first reflector is once again recovered much better.

The reconstructed relative distance exhibits a small bias. The primary reason for this is the relatively sparse discrete representation of the horizons that fails to capture the curvature of a synclinal horizon precisely. Additionally, errors in picking with a particular picking method that deviate from the noise model given in Equation 9 may also result in a bias. The small error bars in the right panels of Figure 2 and 3 show that the velocity-induced uncertainty has been mitigated. Other errors in modeling still may and do affect the reconstructed depth of the horizons. 

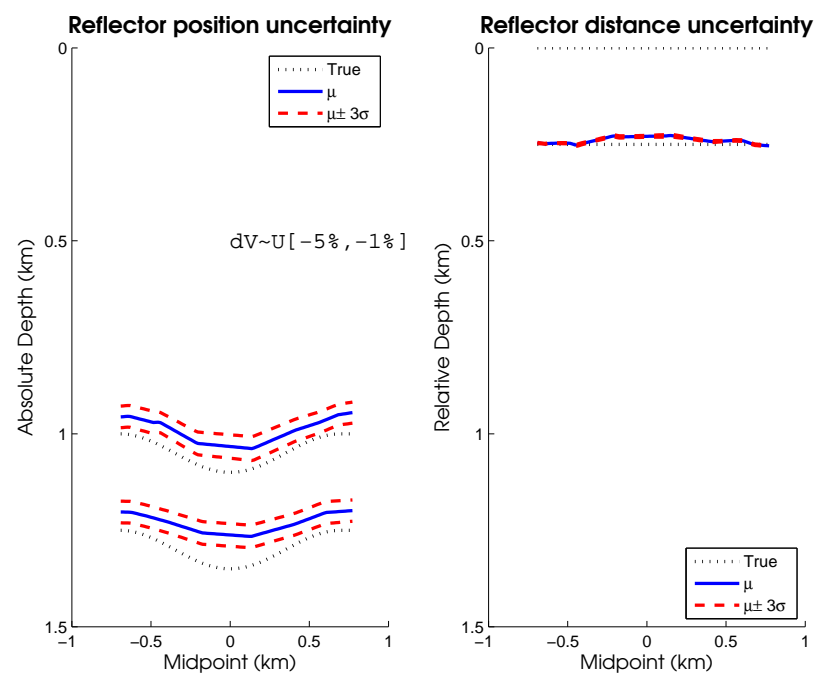

Figure 3: Same as Figure 2 but with $d V \sim$ Uniform $[-5 \%,-1 \%]$.

\section{CONCLUSIONS}

Seismic migration inherently relies on an available velocity model. A good velocity model may not be available, and even the best tomography result will yield an entire set of plausible velocity models. The resulting migrated image should incorporate the uncertainty of the parameters used to build the image. We proposed a Bayesian framework to quantify uncertainty in the migrated images. We considered the effect of velocity uncertainty and the effect of the picking error. Prior information about the velocity model and assumptions about picking allowed us to construct a posterior estimate of the locations of migrated events. This estimate produced not just a single location for each event but it captured the uncertainty in those locations. In some geometries, such as surface seismic and structures with relatively small deviations from horizontal, ray-paths from sources and receivers to different structures largely overlap. This allows for possibility of shared-path cancellation. The effect of the velocity uncertainty along the shared path is limited, which results in better imaging of one structure relative to another.

\section{ACKNOWLEDGEMENTS}

We would like to acknowledge Total for their support. We thank Paul Williamson of Total for fruitful discussions of this project. 
http://dx.doi.org/10.1190/segam2014-1359.1

\section{EDITED REFERENCES}

Note: This reference list is a copy-edited version of the reference list submitted by the author. Reference lists for the 2014 SEG Technical Program Expanded Abstracts have been copy edited so that references provided with the online metadata for each paper will achieve a high degree of linking to cited sources that appear on the Web.

\section{REFERENCES}

Douma, H., and M. V. de Hoop, 2006, Explicit expressions for pre-stack map time-migration in isotropic and VTI media and the applicability of map depth migration in heterogeneous anisotropic media : Geophysics, 71, no. 1, S13-S28, http://dx.doi.org/10.1190/1.2159057.

Glogovsky, V., E. Landa, S. Langman, and T. J. Moser, 2009, Validating the velocity model: The Hamburg score: First Break, 27, no. 3, 77-85.

Grubb, H., A. Tura, and C. Hanitzsch, 2001, Estimating and interpreting velocity uncertainty in migrated images and AVO attributes: Geophysics, 66, 1208-1216, http://dx.doi.org/10.1190/1.1487067.

Osypov, K., M. O’Briain, P. Whitfield, D. Nichols, A. Douillard, P. Sexton, and P. Jousselin, 2011, Quantifying structural uncertainty in anisotropic model building and depth imaging: Hild case study: 73rd Conference \& Exhibition, EAGE, Extended Abstracts, F010.

Poliannikov, O. V., A. E. Malcolm, M. Prange, and H. Djikpesse, 2013, Joint microseismic event location with uncertain velocity: 83rd Annual International Meeting, SEG, Expanded Abstracts, 2094-2098.

Pon, S., and L. R. Lines, 2005, Sensitivity analysis of seismic depth migrations: Geophysics, 70, no. 2, S39-S42, http://dx.doi.org/10.1190/1.1897036.

Yilmaz, Ö., 2001, Seismic data analysis: Processing, inversion and interpretation of seismic data, 2nd ed.: SEG. 